\title{
Gordon Root (Hg.), Schoenberg's Models for Beginners in Composition (= Schoenberg in Words, Bd. 2), New York: Oxford University Press $2016^{1}$
}

Die von dem US-amerikanischen Musiktheoretiker Gordon Root vorgelegte Neuausgabe von Arnold Schönbergs neben der Harmonielehre einzigem zu Lebzeiten erschienenen Lehrwerk ist Teil einer von Sabine Feisst und Severine Neff herausgegebenen neunbändigen Buchreihe der renommierten Oxford University Press mit ausgewählten musiktheoretischen und -analytischen Schriften sowie Korrespondenzen des Komponisten. Bereits erschienen sind auBerdem und ebenfalls jeweils im Jahr 2016 die Bände 5 (Schoenberg's Program Notes and Musical Analyses, hg. von J. Daniel Jenkins) und 8 (Schoenberg's Early Correspondence, hg. von Ethan Haimo und Sabine Feisst).

Schönbergs Models for Beginners in Composition bestehen aus einer umfangreichen, aus langjähriger Unterrichtstätigkeit hervorgegangenen Beispielsammlung nebst erläuterndem "Syllabus" und Glossar. Das Werk, das Rudolf Stephan im Nachwort zu seiner 1972 erschienenen deutschsprachigen Ausgabe als eine von "Schönbergs originellsten Arbeiten ${ }^{2}$ bezeichnet hat, war ursprünglich als Handreichung für die Teilnehmerinnen und Teilnehmer des sechswöchigen Kurses Beginning Composition an der University of California, Los Angeles (UCLA) im Sommer 1942 konzipiert worden, in welchem angehenden Kompositionsstudenten die Grundkenntnisse des kompositorischen Handwerks in komprimierter Form zu vermitteln waren. Zu diesem Zweck hatte Schönberg die von seinem

1 Ich danke Eike Fess vom Arnold Schönberg Center in Wien für freundliche Beantwortung einer Reihe von Fragen, die sich im Zusammenhang mit der kritischen Lektüre des Buchs ergeben haben.
Schüler und Assistenten Leonard Stein handschriftlich kopierten Notenbeispiele im Sommer 1942 vervielfältigen lassen und noch ohne die Erläuterungen und das Glossar über den örtlichen Buchhandel vertrieben $(X)$.

Der Lehrgang beginnt mit elementaren Übungen zur Koordinierung von Melodie und Harmonie auf Grundlage von Zweitaktphrasen und schreitet dann über die Herstellung von Halbsätzen, Sätzen und Perioden zur Bildung von dreiteiligen Formen mit kontrastierendem Mittelteil bis hin zu Menuett und Scherzo fort, und zwar jeweils unter Berücksichtigung sinnvoller harmonischer Verläufe auf Grundlage der erweiterten Tonalität, wie sie Schönberg in seiner Harmonielehre von 1911 und dann noch einmal in dem posthum erschienenen Lehrwerk Structural Functions of Harmony in systematischer Form dargestellt hat. ${ }^{3}$ Die um den Textteil, das Glossar und zahlreiche Beispiele erweiterte Druckfassung des Lehrgangs erschien bei Schönbergs amerikanischem Hauptverlag G. Schirmer Anfang Februar 1943. ${ }^{4}$ Die Ausgabe wurde auf Schönbergs besonderen Wunsch in zwei separaten Heften gedruckt, um eine optimale Handhabbarkeit von Text- und Notenteil zu gewährleisten (XVI). Sie erwies sich aufgrund der überstürzten Drucklegung Schönberg benötigte die Models dringend für seinen Unterricht - jedoch als so fehlerhaft, dass der Verlag einen Errata-Zettel drucken und den noch nicht ausgelieferten Exemplaren beilegen lassen musste. In einer offenbar noch im September desselben Jahres erschienenen NeuSchoenberg 1943. 
auflage wurden dann einige der Fehler korrigiert. $^{5}$

Im Schönberg-Nachlass des Arnold Schönberg Centers in Wien haben sich nicht nur die ursprünglichen Unterrichtsmaterialien, sondern auch mehrere Handexemplare sowohl der im Selbstverlag erschienenen Erstausgabe der Beispielsammlung als auch der beiden gedruckten Schirmer-Ausgaben von 1943 erhalten, in denen Schönberg weitere Korrekturen eingetragen hat. Ein Großteil dieser Korrekturen ist in die revidierte Neuausgabe eingegangen, die Leonard Stein 1972 für den von Schönbergs Erben gegründeten Verlag Belmont Music Publishers vorbereitet hat. ${ }^{6}$ Diese noch heute erhältliche Ausgabe erschien wie die Originalausgabe und die deutsche Übersetzung ${ }^{7}$ im bewährten (Musikalien-)Quartformat.

Der Vorzug der Praktikabilität, der die früheren Ausgaben auszeichnet, wurde bei der Konzeption der vorliegenden Neuausgabe preisgegeben, und zwar sowohl hinsichtlich des zum Spielen der Notenbeispiele auf dem Klavier viel zu kleinen Formats ${ }^{8}$ als auch mit Blick auf die bereits für die revidierte Ausgabe von 1972 vorgenommene Vereinigung der beiden Hefte in einem Buch. Um diese Nach-

5 Dieses Datum geht aus einem der Handexemplare der revidierten Ausgabe (Signatur S142.C2) mit der autographen Aufschrift »revised edition | September 1943 | correcting a few of the | many errors still there« hervor. Diese für die Datierung wichtige Angabe ist im Buch nirgendwo erwähnt. Stattdessen findet sich in einer Anmerkung der nicht weiter begründete Hinweis "Later in 1943 Schirmer reprinted MBC with Schoenberg's corrections" (190, Anm. 18). Auch über eine im Abkürzungs- und Literaturverzeichnis erwähnte weitere Ausgabe aus dem Jahr 1947 gibt Root keinen Aufschluss. Zwar waren die Models der Verlagskorrespondenz zufolge offenbar zwischenzeitlich vergriffen, doch findet sich dort kein Hinweis auf einen Nachdruck.

6 Schoenberg 1972b.

7 Schoenberg 1972a. Dieser Übersetzung liegt nicht die revidierte, sondern die Originalausgabe zugrunde.

8 Zwar wurden die Beispiele neu gesetzt, jedoch unter Beibehaltung der originalen Seitenaufteilung, was eine starke Verkleinerung des Notenbilds zur Folge hat. teile zu kompensieren, wurden auf der Verlagshomepage eigens eingespielte Audiodateien der Notenbeispiele bereitgestellt, die allerdings das für ein ernsthaftes Studium notwendige eigene Spiel nicht ersetzen können. Vor allem aber wurde dem Band eine Fülle von Begleitmaterialien beigegeben, die gut zwei Drittel des Gesamtumfangs ausmachen und einen ausführlichen Kommentar sowie sechs Anhänge umfassen. Letztere beinhalten einen Überblick über die handschriftlichen Quellen einschließlich der Handexemplare (119-130), eine chronologische Aufstellung der von Schönberg unterrichteten Kurse an der UCLA (131-135), eine Auswahl von faksimilierten und kommentierten Hausarbeiten aus dem Kurs Beginning Composition ${ }^{9}$ (137-147), einen Schlüssel zu den von Schönberg verwendeten Symbolen und Abkürzungen (149-152) sowie jeweils eine Einführung (primer) in sein Konzept der >Regionen< (»Regions", 153-165) und der >Alterierungen` (»Transformations», 167187), auf denen die Systematik der Structural Functions of Harmony ${ }^{10}$ beruht. Hinzu kommen als Teil des römisch paginierten Vorspanns mit »Acknowledgements" (XXI-XXIV), »Editorial Notes" (XXV-XXVIII) und »Abbreviations« $(X X I X-X X X I)$ eine als »Preface« bezeichnete kurze Abhandlung der Entstehungsgeschichte der Models (IX-XX) sowie ein umfangreicher Anmerkungsapparat (189-216) ${ }^{11}$, eine Auswahlbibliographie (217-223) und ein Register (225-231).

Angesichts der leichten Verfügbarkeit und der ausgezeichneten Praktikabilität der früheren Ausgaben hat eine konkurrierende Neuausgabe eine ganze Reihe von Voraussetzungen

9 Sie sind Bestandteil der Leonard Stein Collection des Arnold Schönberg Centers, Wien.

10 Schoenberg 1954. Die Übersetzung der originalen englischen Termini beruht auf der von Schönbergs Schüler Erwin Stein übertragenen und herausgegebenen deutschsprachigen Ausgabe (vgl. Schoenberg 1957).

11 Die Anmerkungen sind, wie in angloamerikanischen Buchpublikationen üblich, als Endnoten gesetzt, was die Übersichtlichkeit und Benutzerfreundlichkeit ebenfalls erheblich einschränkt, zumal in der Kopfzeile weder die einzelnen Kapitel noch Seitenbereiche angegeben sind. 
zu erfüllen, um sich als Referenzausgabe behaupten zu können. Sie muss insbesondere angesichts der zwar seit langem angekündigten, aber in absehbarer Zeit nicht realisierbaren Kritischen Gesamtausgabe der Schriften Arnold Schönbergs zwingend eine kritische Ausgabe sein, die einen autoritativen (Noten-)Text auf Grundlage sämtlicher verfügbarer Quellen und mit Hilfe textkritischer Methoden übersichtlich und nachvollziehbar konstituiert. Um die vielfältigen Vorgaben einer derartigen kritischen Ausgabe umzusetzen, ist eine weitgehende lektorierende Betreuung durch den Verlag unumgänglich. Hierzu gehört auch und vor allem, dass die unvermeidbaren Fehler im Neusatz auf ein Minimum beschränkt bleiben, was ein entsprechendes Verlagsengagement bei der Korrekturlesung voraussetzt. Und schließlich muss das ergänzende Material eine historisch-systematische Kontextualisierung der Models bieten, die eine überzeugende Einordnung in Schönbergs musiktheoretisches Werk wie auch in die Theorie- und Lehrtradition des 19. und frühen 20. Jahrhunderts ermöglicht. Leider wird das vorliegende Buch diesen Ansprüchen nicht gerecht.

Obwohl Roots Neuausgabe nicht explizit als 'kritische Ausgabe، bezeichnet ist, ist sie doch erkennbar darum bemüht, die Kriterien einer solchen zu erfüllen. Dies geschieht einerseits durch die Beigabe eines Revisionsberichts (»Editorial Notes", XXV-XXVIII) ${ }^{12}$, andererseits durch die Auflistung und (Kurz-) Beschreibung der herangezogenen Quellen (»Manuscript Sources for $>$ Models for Beginners in Composition«, 119-130). Die Quellen entstammen sechs unterschiedlichen Sammlungen bzw. Konvoluten, die im Arnold Schönberg Center, Wien und -als Depositum des Verlags G. Schirmer - in der Sibley Music Library der Eastman School of Music, Rochester aufbewahrt werden. ${ }^{13}$ Dass die Quellenbeschreibung

12 Bei dem im Inhaltsverzeichnis als »Commentary«, zu Beginn der »Editorial Notes« $(\mathrm{XXV})$ sogar als "Critical Commentary« bezeichneten Kommentarteil handelt es sich nicht um den kritischen Bericht, sondern um den Versuch einer historisch-systematischen Einordnung der Models.

13 Leider spiegeln die in unterschiedlicher Typographie gesetzten Überschriften innerhalb die- in den Anhang verbannt ist, mag auf den ersten Blick als praktikable Entlastung des philologischen Apparats erscheinen. Tatsächlich jedoch offenbart sie einen für die Ausgabe insgesamt charakteristischen Mangel an philologischem Verständnis, da die Festlegung einer Hauptoder Leitquelle üblicherweise die wichtigste Herausgeberentscheidung einer kritischen Ausgabe darstellt. Root dagegen vermeidet eine derartige Festlegung, indem er seiner Edition Leonard Steins Ausgabe von 1972 zugrunde legt, in welche bereits zahlreiche Korrekturen aus den Handexemplaren eingeflossen sind. Eine Aufstellung dieser Änderungen gegenüber der von Schönberg selbst überwachten Ausgabe von 1943 sucht man jedoch vergebens. Stattdessen listet Root in einer Tabelle (»Example 2.1: Editorial changes to the current edition of MBC based on Schoenberg's corrections", XXVI) eine Handvoll weiterer Änderungen und Korrekturen auf, die er den Handexemplaren entnommen hat. Die wichtigste Rolle spielt dabei das Handexemplar mit der Aufschrift »with corrections and remarks for a new edition « (Signatur S142.C3) ${ }^{14}$, aus dem die meisten Korrekturen stammen und das sich daher als Hauptquelle angeboten hätte. Die Tabelle selbst ist unübersichtlich, da die gegenübergestellten Textvarianten als unkommentierte Ausschnitte der originalen Notenbeispiele gesetzt sind, wodurch der Leser genötigt ist, die zumeist minimalen Abweichungen etwa in Gestalt eines ergänzten +-Zeichens oder eines hinzugefügten Warnungsakzidens müh-

ses Abschnitts nicht die Zugehörigkeit der Quellen zu den einzelnen Sammlungen wider, da die Leonard Stein Collection mit ihren drei relevanten Mappen nicht als eigene Sammlung, sondern vermutlich infolge eines Versehens (oder einer unzureichend eingerichteten Vorlage) als Unterkategorie der Sammlung Textbooks for Models for Beginners in Composition ausgewiesen ist. Für den Leser ist die Rekonstruktion des tatsächlichen Quellenzusammenhangs mit einiger Mühe verbunden.

14 In der Tabelle ist die Signatur entsprechend der Aufschrift auf dem Ordner fälschlich als „S143.C3《 angegeben, obwohl Root in der Quellenbeschreibung feststellt, dass es sich bei der auf dem einliegenden Papierstreifen angegebenen Signatur S142.C3 wohl um die »logical catalog number« handele (127). 
sam selbst zu ermitteln. Die Fortsetzung der Tabelle $(X X V I I)^{15}$ - sie findet sich übrigens nicht, wie im Text angegeben, unterhalb, sondern oberhalb des entsprechenden Abschnitts und teilt damit das Schicksal zahlreicher weiterer fehlplatzierter Tabellen und Notenbeispiele im weiteren Verlauf des Buchs - stellt jene Eingriffe zusammen, die der Herausgeber selbst vorgenommen hat. Diese Änderungen beschränken sich im Wesentlichen auf die Ergänzung der von Schönberg nicht ganz konsequent gesetzten horizontalen Durchstreichungen der römischen Stufenziffern im Fall von Alterierungen. Die Entscheidung, die Ziffer III in Beispiel 220 (dritte Fortsetzung, T. 8) durch eine durchgestrichene III zu ersetzen, beruht indes auf einer Fehldeutung, da das originale, als Warnungsakzidens gesetzte \# zu cis ${ }^{1} \mathrm{bzw}$. $\mathrm{cis}^{2}$ in D-Dur leitereigen ist und der fis-Moll-Akkord der III. Stufe somit keine Alterierung darstellt (XXVII, 85 und 208). Die übrigen Eingriffe wie etwa die Tilgung eines überflüssigen Notenschlüssels (73, Bsp. 109) - vermutlich ein Relikt der handschriftlichen Vorlage von Leonard Stein - oder die Ergänzung von Motivbuchstaben (88, Bsp. 228) sind Einzelfälle, die bei konsequenter Vorgehensweise weitere Änderungen hätten nach sich ziehen müssen. Problematisch sind schließlich auch zwei kleinere Korrekturen, die den Textteil des Lehrgangs ("Syllabus «) betreffen. Bei deren Lokalisierung bezieht sich Root nämlich nicht auf seine eigene Ausgabe, sondern auf die Ausgabe von 1972, so dass man die Passage nur mit Glück und nach längerem Suchen findet, wenn man keine Gelegenheit hat, die ältere Ausgabe zurate zu ziehen. Geradezu absurd mutet in diesem Zusammenhang die Korrektur eines in der Vorlage falschen Seitenverweises an, da Root sich auch in diesem Fall auf die Seitenzahl der Ausgabe von 1972 bezieht. Zwar ist der Verweis im Textabdruck skorrekt im Sinn der vorliegenden Neuausgabe als "pages 94-97" angegeben (64), doch hat dies ein eklatantes Miss-

15 Dieser Fortsetzung ist offenbar zur Abgrenzung von der ersten Tabelle die römische Ziffer ii vorangestellt. Da das Äquivalent zur ersten Tabelle fehlt und sich überdies im Text keine Entsprechung findet, entsteht der Eindruck, dass es sich hierbei um ein Relikt aus Roots Typoskript handelt. verhältnis zwischen dem kritischen Apparat und seinem Gegenstand zur Folge, das nur durch eine zusätzliche Anmerkung innerhalb des Textteils (204, Anm. 16) aufgelöst wird. ${ }^{16}$ Überhaupt werden die in der Tabelle aufgelisteten Korrekturen ausnahmslos durch zusätzliche Anmerkungen in den Endnoten abgedeckt, die in den Beispielen durch die vorangestellten Initialen "G.R. « gekennzeichnet sind. ${ }^{17}$ Hier wäre eine Beschränkung entweder auf die Tabelle oder auf die Endnoten ein Gewinn gewesen, da eine Verdopplung der Informationen keinerlei zusätzlichen Erkenntnisgewinn bedeutet, sondern vielmehr zur Verunsicherung des Lesers beiträgt, der an keiner Stelle auf diese Duplizierung hingewiesen wird. Schließlich taugt auch die Tatsache, dass innerhalb der Tabelle selbst sowohl Verdopplungen einzelner Angaben als auch Leerzeilen vorkommen ${ }^{18}$, nicht als vertrauensbildende Maßnahme. Sie offenbart vielmehr ein eklatantes Problem des Buchs in Gestalt einer äußerst mangelhaften Lektorierung.

Das Herzstück des Schönberg'schen Lehrgangs bilden die 249 durchnummerierten und im Textteil kommentierten Notenbeispiele, an die sich in einer Art Anhang 39 weitere Beispiele anschließen, die als Material für weitere Übungen dienen. Außerdem enthält der Notenteil im Anschluss an die Beispiele zum kontrastierenden Mittelteil (Bsp. 233-240) 48 harmonische Schemata, auf deren Grundlage sich ein derartiger Formabschnitt realisieren lässt. Eine Neuausgabe der Models muss sich dementsprechend vor allem daran messen lassen, wie sorgfältig und korrekt die neugesetzten Beispiele Schönbergs originale Vorlagen wiedergeben. Leider vermag die vorliegende Edition

16 Dagegen bezieht sich der von Schönberg selbst stammende Hinweis »motif No. 8 on p. 42 « (63) auf die Ausgabe von 1972. In Roots Ausgabe findet sich das Beispiel dagegen auf Seite 106.

17 Diese Abkürzung wird in einer Anmerkung zu dem analytischen Kommentar innerhalb der »Editorial Notes« (190, Anm. 22) erläutert, die in diesem Zusammenhang allerdings völlig deplatziert ist. In den Beispielen selbst fehlt dagegen ein entsprechender Hinweis.

18 Vgl. XXVI (»Ex. 220, $2^{\text {nd }}$ ending« bzw. »Ex. $220 \ll)$ und XXVII (»Example 225«). 
auch in dieser Hinsicht keineswegs zu überzeugen. Bereits ein oberflächlicher Vergleich mit der Ausgabe von 1972 offenbart eine Vielzahl von Fehlern, die die Korrekturen gegenüber dieser Ausgabe bei weitem - und zwar nicht nur quantitativ, sondern auch qualitativ aufwiegt. Die Fehler in den Beispielen decken von falschem Untersatz über fehlende bzw. überzählige Bögen und Staccatopunkte, falsche Taktartzeichen und Tonartvorzeichen, fehlende Akzidenzien und Noten bis hin zu krassen Tonhöhenfehlern die ganze Bandbreite an Möglichkeiten ab. ${ }^{19}$ Ein besonders peinliches

19 Die folgende Zusammenstellung von Fehlern im Notenteil erhebt keinen Anspruch auf Vollständigkeit: Bsp. 20, T. 1: Falsche (Es-Dur-) Tonartvorzeichnung; Bsp. 30, T. 1: Falscher Untersatz; Bsp. 44: I-III ohne durchgestrichene III (vgl. 205, Anm. 21); Bsp. 61, T. 1: Falscher Untersatz; Bsp. 67, T. 1-2: Falscher Untersatz; Bsp. 75, T. 1 (nach Auftakt): Überzähliges Taktartzeichen; Bsp. 80: I-III-IV statt I-III-VI; Bsp. 153: Taktartzeichen $3 / 4$ statt 2/4; Bsp. 172, T. 1, I. H., 4. Achtel und 3. Viertel: c/g-d/a statt c/a-d/b; Bsp. 174, T. 1, I. H., 4. bis 5. Achtel: aufwärts gehalste Viertelnote $d^{1}$ fehlt; Bsp. 179, T. 2: Bogen endet erst 4. statt 3. Note; Bsp. 191, Auftakt: Achtelnote $d^{1}$ statt $b$; Bsp. 191, T. 1, r. H., 8. Achtel: Überzähliges +Zeichen; Bsp. 199, T. 2, I. H.: $f$ statt f/a abwärts gehalst; Bsp. 217, T. 1, I. H.: 1. Achtel $H$ statt d; Bsp. 221, T. 6-7, r. H., 7./8. Achtel bzw. 3./4. Achtel: Staccatopunkte fehlen; Bsp. 223, T. 5, r. H., 2. und 4. Viertel: Überzählige Staccatopunkte; Bsp. 223, T. 6, r. H., 2. Viertel: Überzähliger Staccatopunkt; Bsp. 224: Taktartzeichen fehlt; Bsp. 226, T. 4, r. H., 4. Achtel: Überzähliger Staccatopunkt zu a ${ }^{1}$; Bsp. 228, T. 5, r. H., 1. Viertel: $\sharp$ (Warnungsakzidens) zu $d^{2}$ fehlt; Bsp. 229, kleines System unter Bsp. 229a: 2. und 3. Taktstrich einfach statt doppelt; Bsp. 237, T. 11, I. H.: Bögen von 5. zu 6. und 7. zu 8. Achtel fehlen; Bsp. 238, T. 10, r. H., 4. Achtel: \# (Warnungsakzidens) zu fis ${ }^{1}$ fehlt; nach Bsp. 240: Schema 35, T. 1, I. H., 3. Viertel: $c^{1} / e^{1}$ auf- statt abwärts gehalst; Schema 37, T. 2, r. H., 1. Halbe: $f^{1} / d e s^{2}$ statt $f^{1} / c^{2}$; Bsp. 242, Alternative e, T. 14-16, I. H.: Bögen von T. 14, 1. bis 3. Viertel, T. 15, 1. bis 2. Viertel und T. 15, 3. Viertel bis T. 16, 1. Viertel fehlen; Bsp. 246, T. 21: Falscher Untersatz der Akkordbezeichnung (2/4 statt 1/4); Bsp. 247, Alternative a, Auftakt zu T. 5, I. H.: Achtel- statt Viertelpause (fehlt in Vorlage); Bsp. 249, T. 18,
Versehen stellt die stufenweise aufsteigende Folge von vier reinen Quinten in Bsp. 172 dar, die Schönberg natürlich nicht geschrieben hat und die einem versierten Musiktheoretiker wie Root bei einer Durchsicht der gesetzten Beispiele nicht hätte entgehen dürfen. Angesichts dieser gravierenden Mängel erscheint die fehlende Sorgfalt bei der grafischen Gestaltung der Beispiele etwa in Gestalt der häufig falschen Halsung einzelner Noten, der teilweise fehlenden Noten- bzw. Hilfslinien (Bsp. 231, 231b) oder auch der uneinheitlichen Schriftgrößen und Stärkegrade bei den numerischen Zusätzen (Zählung der Beispiele, Stufenziffern) geradezu als Petitesse, die gleichwohl kein gutes Licht auf die redaktionelle Betreuung des Buchs (wenn es denn überhaupt eine solche gegeben hat) wirft. Aus demselben Grund wagt man kaum noch zu beanstanden, dass eine Reihe von eindeutigen Fehlern der Ausgabe von 1972 nicht korrigiert wurde. ${ }^{20} \mathrm{Zu}$ diesen gehört unter anderem die auf die dritte Zählzeit des 3/4Taktes gesetzte Halbe Note in der Mittelstimme von Bsp. 242, T. 18, die Root zwar ausführlich diskutiert (209, Anm. 51), aber eben im Notenteil selbst nicht korrigiert, obwohl sein zweiter Lösungsvorschlag (Verschiebung auf die zweite Zählzeit) gegenüber der in der deutschsprachigen Ausgabe von 1972 erfolgten Korrektur (Viertel- statt Halbe Note) klar vorzuziehen ist. ${ }^{21}$

r. H., 3. Achtel: Überzähliger Staccatopunkt (in Vorlage Verschmutzung der Stichplatte); Bsp. 249, T. 28, r. H., 3./4. Achtel: Staccatopunkte fehlen; nach Bsp. 249: Bsp. 5, T. 1, r. H., letzte Note: Falscher Untersatz; Bsp. 17, T. 3, I. H., 2. Viertel: falscher Untersatz; Bsp. 34, T. 7, 5. Achtel: Bogen bis zur folgenden Note fehlt; Bsp. 38, T. 3, 2. Note: Bogen endet erst T. 4, 1. Note statt T. 3, letzte Note.

20 Hierzu zählen etwa die fehlende Halbe Note $g^{1}$ in Bsp. 211, T. 13, die fehlenden Warnungsakzidenzien zu $d$ und $c$ in Bsp. 228, T. 7, die fehlenden $\curvearrowleft$ zu $c^{2}$ bzw. $a^{1}$ in Bsp. 228b, T. 5 bzw. Bsp. 229b, T. 2 oder die fehlende Viertelnote $\mathrm{fis}^{1}$ in Bsp. 230a, T. 6, die bereits in Schoenberg 1972a ergänzt wurde. Auch hätte die auf der ersten Halben Note in Bsp. 48a, T. 2 getilg-

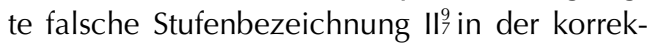
ten Form III⿳亠 ergänzt werden können.

21 Aufgrund der ungünstigen Platzierung des zugehörigen (und aus unerfindlichen Gründen extrem vergrößerten) Notenbeispiels auf der 
Angesichts des verheerenden Eindrucks der Edition des Notenteils stellt sich die Frage, ob wenigstens die Begleitmaterialien einer kritischen Betrachtung standhalten. Im Fokus stehen hier vor allem die Anhänge 5 und 6 sowie der "Commentary", da die Anhänge 2 und 3 (Schönbergs Lehrplan an der UCLA bzw. die mehr oder weniger zufällig überlieferten Hausarbeiten aus dem Sommerkurs Beginning Composition) für sich genommen zwar informativ, aber ohne unmittelbaren Nutzen für den Umgang mit der Ausgabe sind. Die beiden primer in Schönbergs Konzept der Regionen und Alterierungen hingegen sind für Leser, die mit Schönbergs harmonischen Konzepten und speziell mit den Structural Functions of Harmony nicht vertraut sind, durchaus sinnvoll, zumal Schönberg eine derartige verkürzte Einführung in seine Harmonielehre in Gestalt des Praktischen Leitfadens seines Schülers Erwin Stein selbst angeregt hat. ${ }^{22}$ Der Grundgedanke des Konzepts der >Regionen (153-165) beruht auf dem Prinzip der Monotonalität, das in Schönbergs Vorstellung als Analogon zur Zwölftonreihe, die die Einheit einer Komposition garantiert, gesehen werden kann. Ihr Nukleus ist ein hierarchisches Modell in Gestalt einer Matrix, welche die Nähe bzw. Ferne der auf sämtlichen, also auch auf den chromatisch erhöhten oder erniedrigten Stufen gebildeten Akkorde zur Grundtonart schematisch darstellt. In ihrem aus dreimal sechs Akkorden bestehendem Zentrum ist diese Matrix vertikal in Quinten und horizontal in kleinen Terzen sowie durch den Dur-Moll-Wechsel organisiert, was dem nahen Verwandtschaftsverhältnis dieser Stufen Rechnung trägt. In den äußeren Bereichen, d.h. bei den entfernteren Verwandtschaften kommen außerdem Großterzverhältnisse ins Spiel. Da nicht nur sämtliche enharmonisch identischen Töne als eigene Stufen definiert sind, sondern einige Akkorde der entfernteren Regionen in doppelter, in ei-

folgenden Seite (210) wäre auch der unkritische Leser für einen entsprechenden Hinweis dankbar.

22 Stein 1923. Dem ersten primer ist ein knapper Überblick über die Forschungsliteratur zu Schönbergs harmonischen Theoriekonzepten vorangestellt (153-157), der jedoch im Kontext einer Einführung deplatziert wirkt. nem Fall sogar in dreifacher Funktion vorkommen, umfasst die Matrix insgesamt 43 funktional auf die Tonika bezogene Regionen. In den Models wird dieses Konzept in stark reduzierter Gestalt innerhalb des Glossars vorgestellt, wobei die 14 behandelten Regionen für das Verständnis der harmonischen Analysen in den Beispielen vollkommen ausreichen. Dennoch ist die Einführung in das vollständige Konzept zweckmäßig, da die in der Matrix sichtbaren Symmetriebildungen für Schönbergs Denken insgesamt wie auch zumindest für einige seiner Beispiele konstitutiv sind.

Das in Anhang 6 vorgestellte Konzept der 'Alterierungen oder »Transformations" (167187 ) beruht auf der Vorstellung, dass das Repertoire der auf den einzelnen Stufen einer Skala gebildeten Akkorde durch die Einbeziehung von chromatischen iStellvertretern` (zur Herstellung von Zwischendominanten) sowie durch die Entlehnung von Tönen der Molltonika und der Mollsubdominante erweitert werden kann, und bildet so eine wichtige gedankliche Voraussetzung für das übergeordnete Konzept der Monotonalität. Root stellt die wichtigsten derart hergeleiteten Akkordtypen in Gestalt des (nicht leitereigenen) Dominantseptakkords ('Mm7 Transformation`), des halbverminderten $\left(,^{\circ} 7\right.$ Transformation $\left.\iota\right)$ und verminderten Septakkords $\left(\imath^{\circ} 7\right.$ Transformation $\left.<\right)$, des übermäßigen Quintsext- ( $(G e r m a n$ Sixth Transformation ‘) und Terzquartakkords (,French Sixth Transformation «) sowie des neapolitanischen Sextakkords (>Neapolitan Transformation`) vor und analysiert entsprechende Beispiele aus den Models. Die hiermit verbundene Systematisierung trägt zum Verständnis der eher rudimentären harmonischen Analysen innerhalb der Beispiele bei und dürfte insbesondere für den mit Schönbergs Harmoniesystem wenig vertrauten Leser gewinnbringend sein. Etwas fragwürdig ist in diesem Zusammenhang jedoch Roots Versuch, Schönbergs terminologisch nicht weiter differenzierte Bezeichnung einer in seiner Harmonielehre besprochenen Umkehrung des übermäßigen Quintsextakkords mit enharmonisch verwechselter 'None` auf deren Verhältnis zum ausgelassenen Grundton in Gestalt einer übermäßigen Oktave zurückzuführen. Auch wenn sich Root zur Untermauerung seiner These auf das Konzept der `Grundtonerhöhung، hätte berufen 
können, das Schönberg bei seiner Herleitung des Akkords vermutlich von Ernst Friedrich Richter übernommen hat ${ }^{23}$, so steht dem doch entgegen, dass die Akkordbezeichnungen trotz ihrer Herkunft aus der Generalbasspraxis nicht nur für Schönberg, sondern für die meisten Theoretiker am Anfang des 20. Jahrhunderts längst keinen derartigen Bezug mehr hatten. Sie dienten vielmehr lediglich als 'Label für bestimmte Akkordtypen, die in allen möglichen Umkehrungen auftreten konnten. Dies gilt auch für den übermäßigen Quintsextakkord, der seinen Namen zwar in Übereinstimmung mit seiner ursprünglichen Verwendung der übermäßigen Sexte zwischen verminderter Quinte und Terz verdankt, sich jedoch im Lauf des 19. Jahrhunderts von der spezifischen Lagenordnung seiner Töne emanzipiert hat, wobei die übermäßige Sexte eben auch als verminderte Terz in Erscheinung treten konnte. ${ }^{24}$

Mit einem Umfang von 48 Seiten beansprucht der Abschnitt "Commentary" (1-48), bei dem es sich in Wirklichkeit um einen eigenständigen Essay zu den Models handelt, fast ebenso viele Seiten wie Schönbergs gesamter Lehrgang. Zum Einstieg diskutiert Root jene 'Modelle`, die Schönberg seinem Schüler Alban Berg am Anfang von dessen gut dokumentiertem und aufgearbeitetem Kompositionsunterricht im Herbst 1907 an die Hand gab. ${ }^{25}$ Die teils im Faksimile wiedergegebenen Aufzeichnungen beginnen mit einer Tabelle, in der Schönberg systematisch sämtliche Möglichkeiten, einen Viertakter aus zwei bzw. drei Motiven zu bilden, auflistete. Bei seinem Versuch, Schönbergs Vorgehensweise in Regeln zu fassen, unterläuft Root jedoch ein grundsätzlicher Denkfehler (4f.): Seine Regel 1 besagt, dass jede Fortschreitung mit einem Motiv a beginnen müsse. Tatsächlich jedoch sind die Motivbuchstaben durch ihre alphabetische Folge bereits sukzessiv aufeinander bezogen, d.h. Motiv a bezeichnet per se die erste auftretende

23 Vgl. Schönberg 1922, 296; Richter 1905, 87.

24 In einer Fußnote in seiner Harmonielehre thematisiert Schönberg übrigens die sich daraus ergebende fehlende Eindeutigkeit der Bezeichnung des übermäßigen Terzquartakkords (Schönberg 1922, 296).

25 Vgl. Krämer 1996; Berg 2007.
Motivgestalt. Da Root jedoch von absoluten und nicht von relativen Motivgestalten ausgeht, ist er genötigt, eine dritte Regel zu formulieren, die besagt, dass jedes Modell seine einzelnen Bestandteile in alphabetisch ansteigender Reihenfolge bringen müsse, weshalb die Folge a c b a ausgeschlossen sei. Tatsächlich ist diese Folge ausgeschlossen, weil das zweite auftretende Motiv eines derartigen Viertakters grundsätzlich immer mit b bezeichnet ist. Roots Systematisierungsversuch beruht auf der Verkomplizierung eines einfachen Sachverhalts und ist nicht nur unbegründet, sondern sogar irreführend, da er spätestens im Kontext der folgenden Übungen zu Missverständnissen führen würde. Denn hier werden die Buchstaben durch eintaktige Motive ersetzt und zu achttaktigen Sätzchen erweitert, die in harmonischer Hinsicht den in einer weiteren Tabelle zusammengestellten Stufenfolgen Rechnung tragen und auf Motivreihungen beruhen, die Roots >Regeln teils widersprechen. Einige dieser Übungssätzchen sind übrigens als Notenbeispiele wiedergegeben, von denen jedoch kaum eines fehlerfrei übertragen wurde. ${ }^{26}$

Problematisch ist schließlich auch Roots Motivauffassung mit Blick auf die Übungssätzchen. So leitet er beispielsweise das dritte Auftreten der Motivgestalt $b$ in Schönbergs als Beispiel 3.3 wiedergegebenem Beispielsätzchen mittels Umkehrung, Transposition und Einschub aus der ersten Erscheinungsform des Motivs ab. Im Fall der zweiten Motivgestalt ist die Ableitung sogar noch komplizierter (8). Tatsächlich ging Schönberg jedoch von einer nicht intervallisch, sondern rhythmisch determinierten Motivgestalt aus, die überdies mit dem Takt als kleinster Einheit des musikali-

26 Bsp. 3.5, T. 7, r. H., 2. Note: $g^{2}$ statt korrekt $f^{2}$; Bsp. 3.6, T. 2, r. H., 1. Note: $f^{1} / c^{2}$ statt $f^{1}$; I. H., letzte Note: $f / a / d^{1}$ statt $f / a / c^{1} ;$ Bsp. 3.7, T. 4, r. H., 4. Viertel: $f^{1}$ statt $g^{1}$; T. 7, r. H., 1. Note: $a^{1} / c^{2}$ statt $a^{1} ;$ T. 8, r. H., letzte Note: Viertelnote statt Halbe Note. Überdies fehlt ein Hinweis auf den betreffenden Band der Alban Berg Gesamtausgabe, in dem die entsprechenden Seiten aus dem Übungskonvolut faksimiliert und die in Frage stehenden Sätzchen vollständig ediert und kommentiert wurden (vgl. Berg 2007, [XVII] und [XVIII], 5-9 sowie 203-208). 
schen Satzes zusammenfällt. ${ }^{27}$ Zwar spielte selbstverständlich auch schon bei diesen ersten Übungen das für Schönbergs Denken zentrale Variationsprinzip eine Rolle, doch bestimmt es hier weniger das Verhältnis der einzelnen Motive eines Sätzchens als das der Achttakter zueinander. ${ }^{28}$

Im Anschluss an die Diskussion dieser Modellsätzchen aus Bergs Unterricht wendet sich Root den Modellen aus Schönbergs späterer Zeit zu, wobei er sich vor allem auf die ebenfalls auszugsweise faksimilierten und in Übertragungen wiedergegebenen Aufzeichnungen von Gerald Strang bezieht (12-20). Strang gehörte zu Schönbergs ersten Schülern in Los Angeles und hat sich Verdienste als Herausgeber des posthum erschienenen Lehrwerks Fundamentals of Musical Composition erworben. ${ }^{29}$ Zurecht betont Root, dass hier nicht mehr das eintaktige Motiv, sondern die zweitaktige Phrase das kleinste Element der Formbildung darstellt - ein Sachverhalt, der auf einen grundsätzlichen Wandel in Schönbergs Lehrkonzept schließen lässt und der offenbar mit seiner Rezeption der Kompositionslehre von Adolph Bernhard Marx zusammenhängt (14). Dessen Motivbegriff war insofern wesentlich flexibler als die didaktisch motivierte Vereinfachung Lobes, als das Motiv eben nicht als Element des Satzbaus, sondern als taktunabhängiges, variables Tonmaterial aufgefasst wird..$^{30}$ Für Marx war ähnlich wie später für Schönberg die kleinste strukturelle Einheit der musikalischen Konstruktion die zweitaktige, die er - anders als von Root behauptet (20) - sehr wohl mit einem eigenen Terminus bedachte, indem er die beiden Hälften eines viertaktigen Satzes als ,Abschnitte ${ }^{31}$ bezeichnete. Der Terminus ,Phrase` als Synonym für `Zweitakt ‘ findet sich allerdings erst bei Ludwig Bussler, dessen $\mathrm{Mu}$ sikalische Formenlehre eine vereinfachte Bearbeitung des vierbändigen Marx'schen Werks

27 Diese Auffassung beruht auf der Lehrmethode von Johann Christian Lobe (vgl. Lobe 1875, 11, sowie Krämer 1996, 42ff.).

28 Vgl. Krämer 1996, $48 f$.

29 Schoenberg 1967.

30 Vgl. Marx 1846, 34-37; Krämer 1996, 44f.

31 Vgl. Marx 1846, 44; Krämer 1996, 45. für die Unterrichtspraxis darstellt. ${ }^{32}$ Root jedoch führt als möglichen Vermittler nicht Bussler, sondern den Leipziger Komponisten und Musiktheoretiker Stephan Krehl an, der die zweitaktige Einheit in seiner Musikalischen Formenlehre als »kleine[n] Satz « ${ }^{33}$ bezeichnete und der ähnlich wie Schönberg dem Schüler eine Reihe zunehmend chromatisch ausgerichteter harmonischer Modelle für die Konstruktion derartiger Sätze an die Hand gab (22f.). Unerfreulich und leider symptomatisch für das ganze Buch ist, dass sich bei der Wiedergabe der zugehörigen Beispiele erneut entstellende Fehler eingeschlichen haben. So ist der Anfang von Beethovens Hammerklaviersonate op. 106 als "Op. 16" und das Hauptthema des ersten Satzes der Sonate op. 111 wohl aufgrund einer Verwechslung mit der römischen Kardinalzahl III als »Op. 3 « bezeichnet ([24]). ${ }^{34}$

Es folgt eine Diskussion ausgewählter Beispiele aus den Models unter sowohl harmonischen als auch motivischen Gesichtspunkten. Instruktiv ist Roots Analyse des Scherzos D-Dur (Bsp. 247), das hinsichtlich der harmonischen Gestaltung eine auffällige Parallele zu dem gut ein halbes Jahr nach Abschluss des Lehrwerks im Sommer 1943 entstandenen Werk Theme and Variations for Wind Band op. 43a aufweist (34f.). ${ }^{35}$ Diese Beobachtung ist insofern von Interesse, als sie das Potenzial für weitergehende Untersuchungen zum Verhältnis der 'komponierten « Modellsätze in Schönbergs Lehrwerken und seinen Kompositionen aufzeigt. In dieser Hinsicht wäre beispielsweise ein Vergleich der "Period No. 1 « (Bsp. 228) mit dem rhythmisch ähnlichen Hauptthema des ersten Satzes aus dem Vierten Streichquartett loh-

32 Vgl. Bussler 1894, 1: §1 | Zweitakt oder Phrase«; Krämer 1996, 46. Dass Busslers Buch im Schönberg-Kreis bekannt war, geht u.a. daraus hervor, dass es von Berg in seinen beiden Aufsätzen »Warum ist Schönbergs Musik so schwer verständlich?« (Berg 1924a, 330) und »Die musikalischen Formen in meiner Oper 'Wozzeck« (Berg 1924b, 589) zitiert wird.

33 Krehl 1920, 21-29.

34 In zwei weiteren Beispielen auf derselben Seite ist die Opuszahl der Klaviersonate C-Dur op. 2/3 falsch wiedergegeben.

35 Roots Behauptung, op. 43 sei zur gleichen Zeit wie die Models entstanden (34), ist unzutreffend. 
nenswert. Den Abschluss bildet eine Untersuchung des Menuetts B-Dur (Bsp. 242) unter dem Aspekt der auf dem Prinzip der Umkehrung beruhenden symmetrischen harmonischen Fortschreitung, die auch der Matrix der ,Regionen Schaffen insgesamt charakteristisch ist (36-47).

Obwohl der "Commentary" zumindest in seinen analytischen Teilen lesenswert ist, indem er die Bedeutung des Lehrwerks insgesamt unterstreicht und durch die Kontextualisierung neue Sichtweisen eröffnet - die theoriegeschichtliche Einordnung ist dagegen weniger gelungen -, vermag er den insgesamt unerfreu- lichen Gesamteindruck des Buchs, an dem der Herausgeber nicht die Alleinschuld trägt, sondern der zu einem erheblichen Teil auch auf die unzureichende Lektorierung zurückzuführen ist, nicht entscheidend zu verbessern. Dies ist umso misslicher, als hier eine Chance vertan wurde - die Chance nämlich, erstmals ein Schönberg'sches Theoriewerk in einer kritischen Ausgabe vorzulegen, die diese Bezeichnung verdient. Für die Buchreihe insgesamt bleibt zu hoffen, dass die ausstehenden Bände überzeugender geraten.

\section{Ulrich Krämer}

\section{Literatur}

Berg, Alban (1924a), "Warum ist Schönbergs Musik so schwer verständlich?«, Musikblätter des Anbruch 6, 329-341.

Berg, Alban (1924b), „Die musikalischen Formen in meiner Oper 'Wozzeck «", Die Musik 16/5, 587-589.

Berg, Alban (2007), Instrumentalmusik 2. Einzelne Stücke, Variationen, Sonatenentwürfe (= Sämtliche Werke, Bd. II/2.2 [Musikalischer Nachlass. Kompositionen aus der Studienzeit, Teil 2]), Wien: Universal Edition.

Bussler, Ludwig (1894), Musikalische Formenlehre in dreiunddreissig Aufgaben, 2. Auflage, Berlin: Habel.

Krämer, Ulrich (1996), Alban Berg als Schüler Arnold Schönbergs. Quellenstudien und Analysen zum Frühwerk, Wien: Universal Edition.

Krehl, Stephan (1920), Die reine Formenlehre (= Musikalische Formenlehre [Kompositionslehre], Bd. 1), 2. Auflage, Berlin: Vereinigung wissenschaftlicher Verleger.

Lobe, Johann Christian (1875), Von den ersten Elementen der Harmonielehre an bis zur vollständigen Komposition des Streichquartetts und aller Arten von Klavierwerken (= Lehrbuch der musikalischen Komposition, Bd. 1), 4. Auflage, Leipzig: Breitkopf \& Härtel.

Marx, Adolph Bernhard (1846), Die Lehre von der musikalischen Komposition, praktisch- theoretisch, Bd. 1, 3. Auflage, Leipzig: Breitkopf \& Härtel.

Richter, Ernst Friedrich (1905), Lehrbuch der Harmonie. Praktische Anleitung zu den Studien in derselben, 24. Auflage, Leipzig: Breitkopf \& Härtel.

Schönberg, Arnold (1922), Harmonielehre, 3. Auflage, Wien: Universal Edition.

Schönberg, Arnold (1943), Models for Beginners in Composition, New York: Schirmer.

Schönberg, Arnold (1954), Structural Functions of Harmony, hg. von Humphrey Searle, London: Williams and Norgate.

Schönberg, Arnold (1957), Die formbildenden Tendenzen der Harmonie, übersetzt von Erwin Stein, Mainz: Schott.

Schönberg, Arnold (1967), Fundamentals of Musical Composition, hg. von Gerald Strang, London: Faber \& Faber.

Schönberg, Arnold (1972a), Modelle für Anfänger im Kompositionsunterricht, übersetzt und hg. von Rudolf Stephan, Wien: Universal Edition.

Schönberg, Arnold (1972b), Models for Beginners in Composition: Syllabus, Music Examples, and Glossary, Revised Edition, hg. von Leonard Stein, Los Angeles (CA): Belmont.

Stein, Erwin (1923), Praktischer Leitfaden zu Schönbergs Harmonielehre. Ein Hilfsbuch für Lehrer und Schüler, Wien: Universal Edition. 
Krämer, Ulrich (2018): Gordon Root (Hg.), Schoenberg's Models for Beginners in Composition (= Schoenberg in Words, Bd. 2), New York: Oxford University Press 2016. ZGMTH 15/1, 173-182. https://doi.org/10.31751/959

〔C 2018 Ulrich Krämer (kraemer@bbaw.de)

Dieser Text erscheint im Open Access und ist lizenziert unter einer Creative Commons Namensnennung 4.0 International Lizenz.

This is an open access article licensed under a

(c) (i)

Creative Commons Attribution 4.0 International License.

eingereicht / submitted: 10/01/2018

angenommen / accepted: 10/01/2018

veröffentlicht / first published: 30/06/2018

zuletzt geändert / last updated: 03/08/2018 